\title{
A novel function of EpCAM in oral squamous cell carcinoma cells under anchorage-independent conditions
}

\author{
HIROSHI INOUE ${ }^{1,2}$, YUICHI OHNISHI $^{1}$, MASAHIRO NAKAJIMA $^{1}$, KENJI KAKUDO $^{1}$ and MASAMI NOZAKI ${ }^{2}$ \\ ${ }^{1}$ Second Department of Oral and Maxillofacial Surgery, Osaka Dental University, 1-5-17 Otemae, \\ Chuo-ku 540-0008; ${ }^{2}$ Department of Cell Biology, Research Institute for Microbial Diseases, \\ Osaka University, 3-1 Yamadaoka, Suita 565-0871, Osaka, Japan
}

Received May 23, 2011; Accepted June 30, 2011

DOI: 10.3892/ijo.2011.1137

\begin{abstract}
Epithelial cell adhesion molecule (EpCAM), involved in $\mathrm{Ca}^{2+}$-independent homotypic cell-cell adhesion in epithelial tissues, is overexpressed in several cancer types. Although studies investigating the function of EpCAM in cancers have shown that it plays a role in cell proliferation, invasion and metastasis, the overall function of EpCAM in cancer cells has remained elusive. Here, we report a novel function of EpCAM in multicellular aggregates (MCAs). EpCAM inhibition using RNA interference (RNAi) did not affect cell morphology, proliferation or expression of certain genes, including cyclin D1 in monolayer cultures of the human oral squamous cell carcinoma cell lines HSC-3 or HSC-4. However, in HSC-4 cells cultured as MCAs, suppression of EpCAM significantly reduced the expression levels of cyclin D1. Nuclear localization of the cyclin D1 protein was observed in MCAs of HSC-4 cells but not in MCAs of EpCAM knockdown HSC4 cells, suggesting that EpCAM regulates cyclin D1 expression and localization in HSC-4 cells under anchorage-independent conditions. We propose that targeting EpCAM might result in more efficient therapies under certain conditions of oral squamous cell carcinoma.
\end{abstract}

\section{Introduction}

Oral squamous cell carcinoma (OSCC) is the most frequent cancer of the head and neck $(1,2)$ with surgery, radiation, and chemotherapy being the standard treatments. Despite therapeutic advances, functional disorders after surgery often occur. Although many studies investigating molecular targets as markers to determine the prognosis of patients have been developed, further functional analyses are required to develop therapies for oral cancer (3-5).

Correspondence to: Dr Masami Nozaki, Department of Cell Biology, Research Institute for Microbial Diseases, Osaka University, 3-1 Yamadaoka, Suita, Osaka, Japan

E-mail: mnozaki@biken.osaka-u.ac.jp

Key words: oral cancer cell line, EpCAM, cyclin D1, cell proliferation, squamous cell carcinoma, anchorage independence, RNA interference
In independent studies, epithelial cell adhesion molecule (EpCAM) has been observed to be expressed in several carcinomas (6-11). It is a $34-42-\mathrm{kDa}$ transmembrane protein mediating $\mathrm{Ca}^{2+}$-independent homotypic cell-cell adhesion in epithelial cells (12), and does not belong to the four major adhesion molecule families (cadherins, integrins, selectins, and the immunoglobulin superfamily) $(13,14)$. EpCAM consists of an extracellular domain of 265 amino acids including two epidermal growth factor (EGF)-like repeats, a transmembrane domain of 23 amino acids and a short intracellular domain of 26 amino acids (15-17). Expression is restricted to the basolateral membrane of the majority of epithelium tissue, except in adult squamous epithelium and several specific epithelia $(12,17,18)$. EpCAM has been shown to be overexpressed in several carcinomas including colon, lung, stomach, prostate, cervical, and breast cancer (19-22). Furthermore, the expression level of EpCAM reportedly correlates with cell proliferation and invasiveness of cancers including oral cancer, indicating EpCAM could be an efficient molecular target $(19,21,23)$. Clinical studies investgating the efficacy of the monoclonal antibody, catumaxomab, in non-small cell lung cancer and ovarian cancer has indicated the efficacy of targeting EpCAM $(24,25)$.

Cyclin D1 is an important regulator through the G1 phase of the cell cycle, and it forms a cyclin-dependent kinase (CDK) complex by binding Cdk4 and Cdk6 (26). Through phosphorylation and deactivation of retinoblastoma protein by the complex, the E2F transcription factor activates several genes required for advancement from the G1 into the $\mathrm{S}$ phase (27). Uncontrolled cell proliferation is a characteristic of cancer, and high levels of cyclin D1 expression promotes cell growth and malignancy of several cancer types (28-31).

Here we determined that EpCAM expression upregulated or maintained the cell cycle regulator, cyclin D1 in oral cancer cell lines as multicellular aggregates (MCAs).

\section{Materials and methods}

Cell culture. Human OSCC cell lines SAS, HSC-3, and HSC-4 (RIKEN BioResource Center, Ibaraki, Japan) were cultured in Dulbecco's modified Eagle's medium (Mediatech, Inc., Manassas, VA) supplemented with $10 \%$ fetal calf serum (Invitrogen, Carlsbad, CA) at $37^{\circ} \mathrm{C}$ in a humidified atmosphere of $95 \%$ air and $5 \% \mathrm{CO}_{2}$. 
Cell monolayer cultures were prepared by plating on $10-\mathrm{cm}$ cell culture dishes (Asahi Glass, Tokyo, Japan), 6-well plates (Multiwell Plates; Asahi Glass), and 96-well plates (Asahi Glass). To generate MCAs, cells were plated on 35-mm EZ BindShut ${ }^{\circledR}$ culture dishes (Asahi Glass).

Adult mice. Adult mice (3-6 weeks old; ICR male and female; Japan SLC, Inc., Shizuoka, Japan) were maintained according to the institutional guidelines of the Research Institute for Microbial Diseases, Osaka University. The mice were used for RNA isolation from testis, kidney, liver, brain, intestine, stomach, heart, lung, tongue, and ovary.

$R T-P C R$. Total RNA was isolated with TRIzol (Invitrogen) from cultured cells and tissues of the adult mice. cDNA was synthesized using Random Primer 6 (New England BioLabs, Ipswich, MA) and SuperScript ${ }^{\mathrm{TM}}$ III Reverse Transcriptase (Invitrogen). cDNA was amplified by Takara Ex Taq ${ }^{\circledR}$ Hot Start Version (Takara Biotechnology, Shiga, Japan) for PCR analysis. Primer sequences for PCR were EpCAM (Fw, 5'-GA ATGGCTCAAAACTTGGGA; Rv, 5'-ACGCGTTGTGATC TCCTTCT),CyclinD1 (Fw, 5'-CCCTCGGTGTCCTACTTCA; Rv, 5'-GTTTGTTCTCCTCCGCCTCT), GAPDH (Fw, 5'-ACA GTCAGCCGCATCTTCTT;Rv,5'-TGGAAGATGGTGATGG GATT), mEpCAM (Fw, 5'-ATGGACCTGAGAGT GAACGG; Rv, 5'-CACGGCTAGGCATTAAGCTC), mGapdh (Fw, 5'-CC CACTAACATCAAATGGGG;Rv,5'-CCTTCCACAATGCCA AAGTT). Quantification of band intensities were performed with LAS4000 mini (Fujifilm, Osaka, Japan). Expression levels of each sample were normalized against GAPDH mRNA expression.

RNA interference (RNAi). Short interfering RNA (siRNA) treatment was performed using Oligofectamine (Invitrogen) or Lipofectamine 2000 (Invitrogen) according to the manufacturer's instructions. The sequences of siRNAs synthesized by Thermo Fisher Scientific (Waltham, MA) were si-EpCAM, 5'-GUUUA CGGCCAGCUUGUAGdTdT and 5'-CUACAAGCUGGCCG UAAACdTdT; si-scrambled RNA, 5'-UUCUCCGAACGUGU CACGUdTdT and 5'-ACGUGACACGUUCGGAGAAdTdT.

To perform the 3-(4,5-dimethylthiazol-2-yl)-2,5-diphenyltetrazolium bromide (MTT) assay, siRNAs were transfected into HSC-4 cells as monolayers in 96-well plates using Oligofectamine reagent. To perform RT-PCR, siRNAs were transfected into HSC-3 and HSC-4 cells as monolayers in 6-well plates using Lipofectamine 2000 reagent and for HSC-4 cells as MCAs; monolayer cells were transfected using Lipofectamine 2000 and plated on 35-mm EZ BindShut culture dishes.

Cell proliferation assay. Proliferation of siRNA transfected cells after incubation for $0,24,48$, or $96 \mathrm{~h}$ in 96 -well plates was assessed using the MTT assay (CellTitler $96{ }^{\circledR}$ Non-Radioactive Cell Proliferation Assay; Promega, Tokyo, Japan) according to the manufacturer's instructions. The absorbance at $570 \mathrm{~nm}$ was measured using a 96-well plate reader (Labsystems Multiskan MS-UV; Labsystems, Helsinki, Finland).

Immunofluorescence staining. MCA cultures of HSC-4 cells were fixed with $3.5 \%$ formaldehyde, permeabilized with $0.2 \%$ Triton X-100, and blocked with Image-iT ${ }^{\mathrm{TM}}$ FX Signal Enhancer

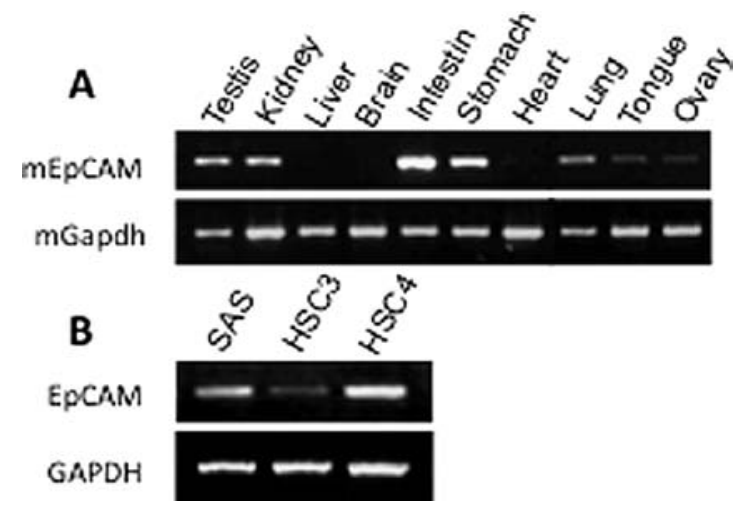

Figure 1. EpCAM expression in mouse tissues and human oral cancer cell lines. (A) EpCAM expression in the tissues of adult mice; and (B) EpCAM expression in SAS, HSC-3, and HSC-4 cells were analyzed by RT-PCR.

(Invitrogen). Cyclin D1 antibody was used as primary antibody. Next, Alexa488 conjugated IgG (Invitrogen) was used as secondary antibodies. After incubation with the antibodies, SlowFade ${ }^{\circledR}$ Gold antifade reagent with DAPI (Invitrogen) was added and coverslips mounted. The specimens were observed using an immunofluorescence microscope (Leica Microsystems Japan, Tokyo, Japan).

Statistical analysis. A Mann-Whitney U test was performed using StatView 5.0 (Abacus Concepts Inc., Berkeley, CA) to assess the statistically significance difference between samples. Data are presented as the mean $\pm \mathrm{SD}$. P-values $<0.05$ were considered statistically significant.

\section{Results}

EpCAM expression associates with oncogenesis in the tongue. To confirm the association of EpCAM expression with oncogenesis in the tongue, we analyzed EpCAM mRNA levels in mouse tissues and human oral cancer cell lines using RT-PCR. EpCAM mRNA was clearly detected in testis, kidney, intestine, and lung of mice, and faintly in the tongue and ovary (Fig. 1A). In oral cancer cell lines derived from tongue, EpCAM mRNA was strongly detectable in SAS and HSC-4 cells and weakly in HSC-3 (Fig. 1B), indicating that the expression levels of EpCAM were different depending on the locus of derived tissues and differentiation of cells. These data suggest that EpCAM expression associates with carciogenesis in the tongue.

Effects of downregulation of EpCAM in oral cancer cell lines as monolayers. Previous studies suggest that EpCAM is related to the prolifration and invasion of cancer cells $(19,21,23)$. To confirm the function of EpCAM on proliferation in oral cancer cell lines as monolayers, EpCAM knockdown in HSC-3 and HSC-4 cells by transient transfection with EpCAM siRNA was performed. EpCAM mRNA levels decreased drastically in the siRNA transfected cells (Fig. 2B and C). In the control cells transfected with or without scrambled RNA, the expression level of EpCAM was not affected (Fig. 2B and C). Downregulation of EpCAM did not significantly influence HSC-4 cell proliferation (Fig. 2A) or cyclin D1 expression (Fig. 2B). Downregulation of EpCAM in HSC-3 cells did not affect the expression level of cyclin D1 

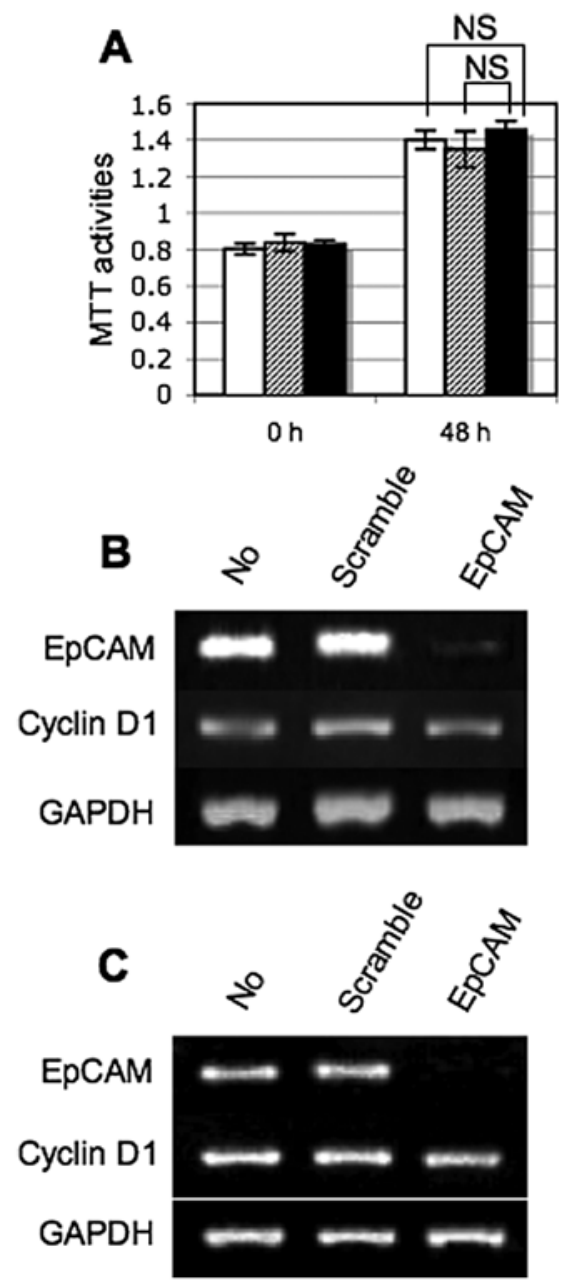

Figure 2. Effects of EpCAM siRNA on HSC-3 and HSC-4 monolayers. (A) HSC-4 cells plated on 96-well plates of $5 \times 10^{3}$ cells per well. After incubation for $48 \mathrm{~h}$, activity of HSC-4 cells transfected with (shaded column) or without (white column) scrambled siRNA $(200 \mathrm{nM})$ or transfected with EpCAM siRNA $(200 \mathrm{nM})$ (black column) were determined using MTT assay. Data are presented as the mean $\pm \mathrm{SD}$ from four independent experiments (Mann-Whitney U test, no-siRNA vs scramble siRNA and no-siRNA vs EpCAM siRNA; NS, not significant). (B) HSC-4 cells were plated on 6 -well plates of $1 \times 10^{5}$ cells per well, and transfected with scrambled siRNA ( $200 \mathrm{nM})$ or EpCAM siRNA (200 $\mathrm{nM})$. After $24 \mathrm{~h}$, cells were analyzed for EpCAM and cyclin D1 mRNA by RT-PCR. Representative results are shown from two independent experiments. (C) HSC-3 cells plated on 6-well plates of $1 \times 10^{5}$ cells per well and transfected with siRNAs. EpCAM and cyclin D1 mRNA were analyzed by RT-PCR. Representative results are shown from two independent experiments.

(Fig. 2C). These results indicate that EpCAM expression did not impact cell proliferation and cyclin D1 expression level in HSC-3 and HSC-4 cells as monolayers.

Effects of downregulation of EpCAM on cyclin D1 level in oral cancer cell line as MCAs. To investigate the role of EpCAM in oral cancer cell lines under anchorage-independent conditions, transfection of siRNA was carried out using Lipofectamine 2000 in HSC-4 cells as MCAs. Suspension culture of HSC-4 cells transfected with siRNA in non-adhesive culture dishes (EZ BindShut) formed MCAs indistinguishable from the control MCAs (data not shown). Dramatic reduction of the EpCAM mRNA level in EpCAM siRNA transfected MCAs was detected by RT-PCR (Fig. 3A and B). Data suggest that
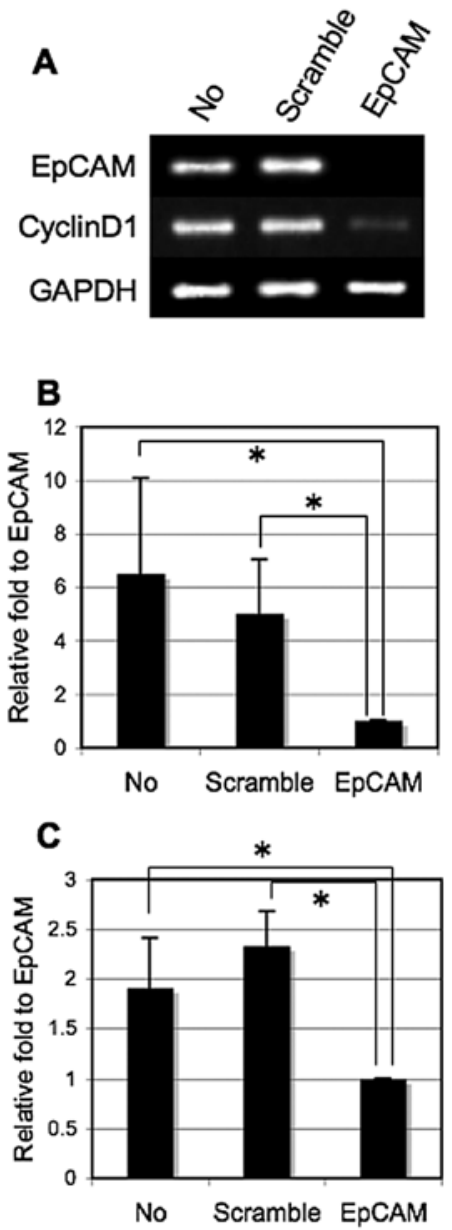

Figure 3. Effects of EpCAM siRNA on HSC-4 cells as MCAs. (A) siRNA transfected HSC-4 cells as MCAs were plated on $35 \mathrm{~mm}$ EZ BindShut culture dishes of $2 \times 10^{5}$ cells. After $24 \mathrm{~h}$, cells were analyzed for EpCAM and cyclin D1 level by RT-PCR. Representative results are shown from three independent experiments. (B) EpCAM/GAPDH intensities are the mean $\pm \mathrm{SD}$ from three independent experiments (Mann-Whitney $\mathrm{U}$ test; * $\mathrm{P}<0.05$ ). (C) The cyclin D1/GAPDH intensities are the mean values with SD from three independent experiments (Mann-Whitney U test; ${ }^{*} \mathrm{P}<0.05$ ).

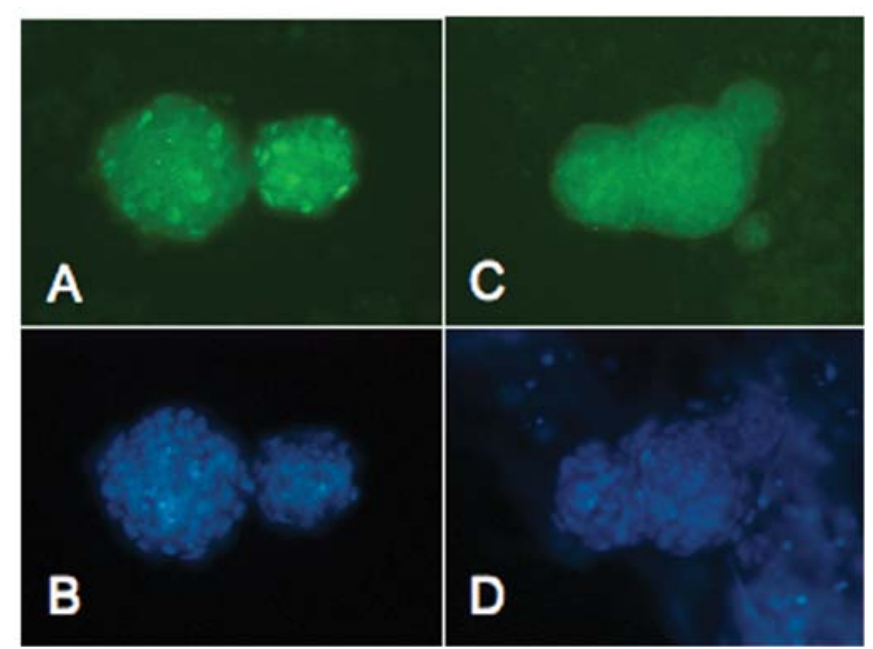

Figure 4. Localization of Cyclin D1 protein in HSC-4 cells as MCAs culture. Control (A and B) or siRNA transfected (C and D) HSC-4 cells as MCAs were plated on $35 \mathrm{~mm}$ EZ-BindShut culture dishes. After $48 \mathrm{~h}$, localization of Cyclin D1 was assessed by immunofluorescent staining (A and C). Nuclei were counterstained with DAPI (B and D). Results are representative of at least three independent experiments. 
cell surface EpCAM molecules are not involved with cell aggregation. Cyclin D1 expression in EpCAM downregulated MCAs was significantly repressed (Fig. 3A and C), indicating that the EpCAM expression could be associated with the cyclin D1 expression in MCAs of HSC-4 cells.

To examine the localization of cyclin D1 in oral cancer cell lines as MCAs, immunofluorescence staining was performed using cyclin D1-specific antibody. Specific staining was distinctly localized to the nucleus in some cells in control MCAs (Fig. 4A). On the other hand, nuclear signals of cyclin D1 staining could not be detected in MCAs from HSC4 cell-transfected siRNA for EpCAM (Fig. 4C). These results show that nuclear restricted cyclin D1 protein localization disappeared in EpCAM eliminated MCAs of HSC4 cells.

\section{Discussion}

The expression patterns of EpCAM in normal tissues of mice and in human OSCC cell lines suggest that EpCAM levels increase during carcinogenesis from the tongue and their overexpression was maintained in parts of tumor tissues because its expression is highly conserved between humans and mice $(14,32)$. This is consistent with previous studies reporting that EpCAM expression is related to cell proliferation in several cancers through the regulation of specific genes including cell cycle regulators $(19,21,23,33)$. Although EpCAM did not contribute to cell proliferation in HSC-4 and HSC-3 cells as monolayers, RNAi-mediated downregulation of EpCAM expression reduced the mRNA expression level of cyclin D1 in MCAs and eliminated cyclin D1 protein in nuclei of MCAs. These data indicate that cell proliferation through cyclin D1 expression in HSC-4 cells is regulated by EpCAM under anchorage-independent cell aggregation conditions. Thus, EpCAM plays various roles depending on the environment of the surrounding cells as well as cell types.

EpCAM is known to be a marker of poor prognosis including metastasis in several carcinomas (34-38). Metastasis is a multistep process, during which primary tumor cells invade adjacent tissues, intravasate, translocate through the vasculature, arrest in distant capillaries, extravasate into the surrounding tissue, and finally proliferate into second tumors (39). Metastatic cancer cells need anoikis resistancy and growth ability in single cells and small cell clusters that are anchorage-independent. MCAs represent a culture model of anchorage-independence (40) and HSC-4 cells with or without siRNA treatment for EpCAM exhibited survival in MCAs culture, indicating that HSC-4 cells have the ability of anoikis resistance independent of EpCAM. Thus, EpCAM may assist cancer cells to initiate growth in a small clusters losing anchoradge-dependency in delivery through the lymphatic and circulatory systems or arrest around the target tissue.

EpCAM, one of the most highly and frequently expressed tumor-associated antigens, is found on a wide range of epithelial cancers (22). Furthermore, EpCAM was identified as a cancer stem cell antigen (41-43). It has also been highlighted as a target antigen for cancer immunotherapy, and many antibody-based therapeutic approaches targeting EpCAM are currently in development (44-49). Alternatively, the possibility of RNAibased therapeutics has been reported (50). We successfully applied RNA silencing to inhibit the expression of EpCAM, thereby decreasing cyclin D1 levels in cancer cells. Therefore, we propose the possibility that RNAi-mediated gene silencing of EpCAM may be a useful strategy for treatment of tongue cancer metastasis.

\section{References}

1. Lippman SM, Sudbø J and Hong WK: Oral cancer prevention and the evolution of molecular-targeted drug development. J Clin Oncol 23: 346-356, 2005.

2. Kato Y, Uzawa K, Yamamoto N, et al: Overexpression of Septin1: possible contribution to the development of oral cancer. Int J Oncol 31: 1021-1028, 2007.

3. Alevizos I, Mahadevappa M, Zhang X, et al: Oral cancer in vivo gene expression profiling assisted by laser capture microdissection and microarray analysis. Oncogene 20: 6196-6204, 2001.

4. Macabeo-Ong M, Shiboski CH, Silverman S, et al: Quantitative analysis of cathepsin L mRNA and protein expression during oral cancer progression. Oral Oncol 39: 638-647, 2003.

5. Muramatsu T, Saitoh M, Ro Y, et al: Inhibition of syndecan-1 expression and function in oral cancer cells. Oncol Rep 20: 1353-1357, 2008.

6. Edwards DP, Grzyb KT, Dressler LG, et al: Monoclonal antibody identification and characterization of a $\mathrm{Mr}$ 43,000 membrane glycoprotein associated with human breast cancer. Cancer Res 46: 1306-1317, 1986.

7. Momburg F, Moldenhauer G, Hämmerling GJ and Möller P: Immunohistochemical study of the expression of a $\mathrm{Mr} 34,000$ human epithelium-specific surface glycoprotein in normal and malignant tissues. Cancer Res 47: 2883-2891, 1987.

8. Perez MS and Walker LE: Isolation and characterization of a cDNA encoding the KS1/4 epithelial carcinoma marker. J Immunol 142: 3662-3667, 1989.

9. Strnad J, Hamilton AE, Beavers LS, et al: Molecular cloning and characterization of a human adenocarcinoma/epithelial cell surface antigen complementary DNA. Cancer Res 49: 314-317, 1989.

10. Simon B, Podolsky DK, Moldenhauer G, Isselbacher KJ, GattoniCelli S and Brand SJ: Epithelial glycoprotein is a member of a family of epithelial cell surface antigens homologous to nidogen, a matrix adhesion protein. Proc Natl Acad Sci USA 87: 2755-2759, 1990.

11. Szala S, Froehlich M, Scollon M, et al: Molecular cloning of cDNA for the carcinoma-associated antigen GA733-2. Proc Natl Acad Sci USA 87: 3542-3546, 1990.

12. Litvinov SV, Velders MP, Bakker HA, Fleuren GJ and Warnaar SO: Ep-CAM: a human epithelial antigen is a homophilic cell-cell adhesion molecule. J Cell Biol 125: 437-446, 1994.

13. Litvinov SV, Balzar M, Winter MJ, et al: Epithelial cell adhesion molecule (Ep-CAM) modulates cell-cell interactions mediated by classic cadherins. J Cell Biol 139: 1337-1348, 1997.

14. Balzar M, Prins FA, Bakker HA, Fleuren GJ, Warnaar SO and Litvinov SV: The structural analysis of adhesions mediated by Ep-CAM. Exp Cell Res 246: 108-121, 1999.

15. Balzar M, Bakker HA, Briaire-De-Bruijn IH, Fleuren GJ, Warnaar SO and Litvinov SV: Cytoplasmic tail regulates the intercellular adhesion function of the epithelial cell adhesion molecule. Mol Cell Biol 18: 4833-4843, 1998.

16. Balzar M, Briaire-de Bruijn IH, Rees-Bakker HA, et al: Epidermal growth factor-like repeats mediate lateral and reciprocal interactions of Ep-CAM molecules in homophilic adhesions. Mol Cell Biol 21: 2570-2580, 2001.

17. Winter MJ, Nagtegaal ID, van Krieken JH and Litvinov SV: The epithelial cell adhesion molecule (Ep-CAM) as a morphoregulatory molecule is a tool in surgical pathology. Am J Pathol 163: 2139-2148, 2003.

18. De Boer CJ, van Dorst E, van Krieken H, et al: Changing roles of cadherins and catenins during progression of squamous intraepithelial lesions in the uterine cervix. Am J Pathol 155: 505-512, 1999.

19. Litvinov SV, van Driel W, van Rhijn CM, et al: Expression of Ep-CAM in cervical squamous epithelia correlates with an increased proliferation and the disappearance of markers for terminal differentiation. Am J Pathol 148: 865-875, 1996.

20. Gastl G, Spizzo G, Obrist P, Dünser M and Mikuz G: Ep-CAM overexpression in breast cancer as a predictor of survival. Lancet 356: 1981-1982, 2000. 
21. Osta WA, Chen Y, Mikhitarian K, et al: EpCAM is overexpressed in breast cancer and is a potential target for breast cancer gene therapy. Cancer Res 64: 5818-5824, 2004.

22. Went P, Vasei M, Bubendorf L, et al: Frequent high-level expression of the immunotherapeutic target Ep-CAM in colon, stomach, prostate and lung cancers. Br J Cancer 94: 128-135, 2006

23. Yanamoto S, Kawasaki G, Yoshitomi I, Iwamoto T, Hirata $\mathrm{K}$ and Mizuno A: Clinicopathologic significance of EpCAM expression in squamous cell carcinoma of the tongue and its possibility as a potential target for tongue cancer gene therapy. Oral Oncol 43 : 869-877, 2007.

24. Burges A, Wimberger P, Kümper C, et al: Effective relief of malignant ascites in patients with advanced ovarian cancer by a trifunctional anti-EpCAM $\mathrm{x}$ anti-CD3 antibody: a phase $\mathrm{I} / \mathrm{II}$ study. Clin Cancer Res 13: 3899-3905, 2007.

25. Sebastian M, Passlick B, Friccius-Quecke H, et al: Treatment of non-small cell lung cancer patients with the trifunctional monoclonal antibody catumaxomab (anti-EpCAM x anti-CD3): a phase I study. Cancer Immunol Immunother 56: 1637-1644, 2007.

26. Sherr CJ: G1 phase progression: cycling on cue. Cell 79: 551-555, 1994.

27. Weinberg RA: The retinoblastoma protein and cell cycle control. Cell 81: 323-330, 1995.

28. Sherr CJ: Cancer cell cycles. Science 274: 1672-1677, 1996

29. Arber N, Doki Y, Han EK, et al: Antisense to cyclin D1 inhibits the growth and tumorigenicity of human colon cancer cells. Cancer Res 57: 1569-1574, 1997.

30. Tashiro E, Tsuchiya A and Imoto M: Functions of cyclin D1 as an oncogene and regulation of cyclin D1 expression. Cancer Sci 98 629-635, 2007.

31. Fernando R, Foster JS, Bible A, et al: Breast cancer cell proliferation is inhibited by BAD: regulation of cyclin D1. J Biol Chem 282: 28864-28873, 2007.

32. Bergsagel PL, Victor-Kobrin C, Timblin CR, Trepel J and Kuehl WM: A murine cDNA encodes a pan-epithelial glycoprotein that is also expressed on plasma cells. J Immunol 148: 590-596, 1992.

33. Spizzo G, Went P, Dirnhofer S, et al: High Ep-CAM expression is associated with poor prognosis in node-positive breast cancer Breast Cancer Res Treat 86: 207-213, 2004.

34. Stoecklein NH, Siegmund A, Scheunemann P, et al: Ep-CAM expression in squamous cell carcinoma of the esophagus: a potential therapeutic target and prognostic marker. BMC Cancer 6: 165,2006

35. Kimura H, Kato H, Faried A, et al: Prognostic significance of EpCAM expression in human esophageal cancer. Int J Oncol 30 : $171-179,2007$.
36. Dupont VN, Gentien D, Oberkampf M, De Rycke Y and Blin N: A gene expression signature associated with metastatic cells in effusions of breast carcinoma patients. Int J Cancer 121: 1036-1046, 2007.

37. Du W, Ji H, Cao S, et al: EpCAM: a potential antimetastatic target for gastric cancer. Dig Dis Sci 55: 2165-2171, 2009.

38. Fidler IJ: The pathogenesis of cancer metastasis: the 'seed and soil' hypothesis revisited. Nat Rev Cancer 3: 453-458, 2004.

39. Humtsoe JO and Kramer RH: Differential epidermal growth factor receptor signaling regulates anchorage-independent growth by modulation of the PI3K/AKT pathway. Oncogene 29: 1214-1226, 2010.

40. Maetzel D, Denzel S, Mack B, et al: Nuclear signalling by tumour-associated antigen EpCAM. Nat Cell Biol 11: 162-171, 2009.

41. Al-Hajj M, Wicha MS, Benito-Hernandez A, Morrison SJ and Clarke MF. Prospective identification of tumorigenic breast cancer cells. Proc Natl Acad Sci USA 100: 3983-3988, 2003.

42. O'Brien CA, Pollett A, Gallinger S and Dick JE: A human colon cancer cell capable of initiating tumour growth in immunodeficient mice. Nature 445: 106-110, 2007.

43. Li C, Heidt DG, Dalerba P, et al: Identification of pancreatic cancer stem cells. Cancer Res 67: 1030-1037, 2007.

44. Punt CJ, Nagy A, Douillard JY, et a: Edrecolomab alone or in combination with fluorouracil and folinic acid in the adjuvant treatment of stage III colon cancer: a randomised study. Lancet 360: 671-677, 2002.

45. Ammons WS, Bauer RJ, Horwitz $\mathrm{AH}$, et al: In vitro and in vivo pharmacology and pharmacokinetics of a human engineered monoclonal antibody to epithelial cell adhesion molecule. Neoplasia 5: 146-154, 2003.

46. Goel S, Bauer RJ, Desai K, et al: Pharmacokinetic and safety study of subcutaneously administered weekly ING-1, a human engineered monoclonal antibody targeting human EpCAM, in patients with advanced solid tumors. Ann Oncol 18: 1704-1707, 2007.

47. Oberneder R, Weckermann D, Ebner B, et al: A phase I study with adecatumumab, a human antibody directed against epithelial cell adhesion molecule, in hormone refractory prostate cancer patients. Eur J Cancer 42: 2530-2538, 2006.

48. Naundorf S, Preithner S, Mayer P, et al: In vitro and in vivo activity of MT201, a fully human monoclonal antibody for pancarcinoma treatment. Int J Cancer 100: 101-110, 2002.

49. Shen J and Zhu Z: Catumaxomab, a rat/murine hybrid trifunctional bispecific monoclonal antibody for the treatment of cancer. Curr Opin Mol Ther 10: 273-284, 2008.

50. Gondi CS and Rao JS: Concepts in in vivo siRNA delivery for cancer therapy. J Cell Physiol 220: 285-291, 2009. 